Town. One of the MSM reported a persistent urethral discharge which had failed to respond to previous therapy with oral cefixime. Agar dilution minimum inhibitory concentration assays were performed for eight antibiotics. The Johannesburg patients' isolates were further characterised by identification of key $\beta$-lactamassociated resistance mutations in $p e n A, m t r R$ and its promoter, porB 1b, ponA, and pilQ through PCR-based amplification and DNA sequencing. For molecular epidemiological characterisation, all three isolates were typed by $N$. gonorrhoeae multi-antigen sequencing typing (NG-MAST); additionally, full-length porB gene sequencing and multi-locus sequence typing (MLST) were performed for the Johannesburg isolates.

Results All three isolates were resistant to cefixime, ciprofloxacin, penicillin and tetracycline, intermediate/resistant to azithromycin but susceptible to ceftriaxone and gentamicin. The Johannesburg isolates had the type XXXIV penA mosaic allele in addition to previously described resistance mutations in the $m t r R$ promoter (A deletion), porB1b (penB) (G101K, A102N) and ponA1 (L421P). All three isolates had an identical $N$. gonorrhoeae multi-antigen sequence type (ST4822). The two Johannesburg isolates had an identical multilocus sequence type (ST1901).

Conclusions All three strains were resistant to cefixime and were epidemiologically linked with identical NG-MAST sequence types. The Johannesburg isolates possessed a number of key $\beta$-lactamassociated resistance mutations and the type XXXIV penA mosaic allele. These two isolates belonged to a successful international MSM-linked multi-drug-resistant gonococcal clone (MLST ST1901), associated with several cefixime treatment failures in Europe and North America.

\section{P1.021 A NATIONAL STUDY UTILISING THE SEQUENOM MASSARRAY IPLEX PLATFORM FOR HIGH THROUGHPUT MLST-BASED TYPING AND CHARACTERISATION OF RESISTANCE MECHANISMIS IN NEISSERIA GONORRHOEAE}

doi:10.1136/sextrans-2013-051184.0242

${ }^{1}$ E Trembizki, ${ }^{2} \mathrm{M}$ Chen, ${ }^{3 B}$ Donovan, ${ }^{2,4} \mathrm{C}$ Fairley, ${ }^{3} \mathrm{R}$ J Guy, ${ }^{3} \mathrm{~J} \mathrm{M}$ Kaldor, ${ }^{5} \mathrm{M}$ M Lahra, ${ }^{3} \mathrm{D}$ G Regan, ${ }^{1,6} \mathrm{~J}$ Ward, 'D Whiley on behalf of the GRAND study investigators. ${ }^{1} \mathrm{OPID}$ Laboratory, OCMRI, the University of Queensland, Brisbane, Australia; ${ }^{2}$ Melbourne Sexual Health Centre, Carlton Victoria, Australia; ${ }^{3}$ The Kirby Institute, University of New South UK, Sydney, Australia; ${ }^{4}$ Melbourne School of Population and Global Health, University of Melbourne, Melbourne, Australia; ${ }^{5}$ WHO Collaborating Centre for STD and HIV, Microbiology Department, SEALS, Prince of UK Hospital, Sydney, New South UK, Australia; ${ }^{6}$ Baker IDI, Central Australia, Australia

Introduction Strain-typing and characterisation of associated resistance mechanisms is pivotal to understanding the development and spread of Neisseria gonorhoeae (NG) antimicrobial resistance (AMR). In Australia, we have embarked on a national study to determine the molecular basis of AMR in our local isolates with a view to implementing broad-based molecular surveillance for NG AMR.

Methods In this initial phase of the study, called GRAND (Gonorrhoea Resistance Assessment via Nucleic acid Detection), we are using the Sequenom MassARRAY iPLEX MALDI-TOF MS platform to characterise all available isolates $(n=2373)$ collected throughout Australia in the first half of 2012. To date, two iPLEX methods have been developed and validated: (1) a typing method targeting 14 informative SNPs previously shown to predict an MLST type; and (2) an AMR method targeting 11 common mutations associated with $\mathrm{N}$. gonorrhoeae resistance to penicillin, ciprofloxacin, azithromycin and ceftriaxone, including important mutations on the penicillin binding protein (PBP2): A501 substitutions and the mosaic PBP2 sequence.

Results The results to date show that the technology is well suited for high-throughput typing of N. gonorhoeae isolates. In particular, we found it can be used on heat-denatured isolates (removing the need for a commercial DNA extraction kit) and can genotype (using both iPLEX reactions) up to 384 isolates within one working day for less than \$AUS20.00 (€15.00) per isolate.

Conclusions The data from this study will provide pivotal information to inform the implementation of molecular-based NG AMR surveillance. Validation and testing is ongoing.

\section{P1.022 HUMAN PAPILLOMAVIRUS 16 VARIANTS ANALYSIS IN MULTIPLE INFECTIONS}

doi:10.1136/sextrans-2013-051184.0243

${ }^{1} \mathrm{~N}$ Fontecha, ${ }^{1} \mathbf{M}$ Basaras, ${ }^{1} \mathrm{E}$ Arrese, ${ }^{2} \mathrm{~S}$ Hernáez, ${ }^{2} \mathrm{D}$ Andía, ${ }^{2} \mathrm{~V}$ Esteban, ${ }^{1,2} \mathrm{R}$ Cisterna. ${ }^{1}$ University of Basque Country, Leioa, Spain; ${ }^{2}$ Basurto Hospital, Bilbao, Spain

Background/Objectives Human papillomavirus type 16 (HPV $16)$ is the primary aetiology of cervical cancer.

Risk factors associated to develop of malignant lesions include: infection persistence, specific HPV 16 variants and multiple infections presence.

We had characterised the genomic variability of E6, E7 and L1 genes in HPV 16 multiple infection patients samples and analysed the relationship between intratypic variants and lesion grade.

Methods HPV 16 multiple infection samples were amplified with three region type-specific primers and amplicons were sequenced using the "Big Dye Terminator Cycle Sequencing kit".

Sequences were aligned using Edit Sequence Alignment Editor and ClustalW, and compared with Genbank reported reference sequences: European (E), African (AF1 and AF2) and Asian-American (AA).

Lesions were divided as negative, low-grade (L-SIL) or high-grade (H-SIL).

Results HPV 16 multiple infections were identified in 125 samples and 78 of them were analysed for intratypic variations: $72 \mathrm{E}$ variants $(92.3 \%), 4$ AA variants $(5.1 \%)$, one AF1 (1.3\%) and one AF2 variant $(1.3 \%)$

In E6 region, missense mutations (A104del and T350G) were defined in $59 \%$ and $41 \%$ of samples. In $\mathrm{E} 7$ region, a mainly synonymous variation (G849A, 41.33\%) was detected. In L1 region, nonsynonymous replacements were only identified: 6901insCAT (30\%), 6902 insATC (65.7\%) and GAT6951del (97.1\%).

European variants were mainly detected in samples with no lesion while non-european variants were only found in H-SIL or L-SIL.

Conclusions E6, E7 and L1 genes are useful to determinate among E, AA and AF1/AF2 variants. Non-european variants are also present in our population.

Nucleotide variations different to define variants must be studied owing to their potential impact on pathogenesis. T350G nucleotide substitution is associated with elevated risk of cervical carcinomas. These variations should be taken into consideration.

Funding: S-PC11BF002 project (Saiotek, Department of Industry, Basque Government).

\section{P1.023 MOLECULAR TYPING OF TREPONEMA PALLIDUM FROM AN ONGOING SYPHILIS OUTBREAK IN DENMARK}

doi:10.1136/sextrans-2013-051184.0244

${ }^{1}$ K Salado-Rasmussen, ${ }^{2} \mathrm{~T}$ L Katzenstein, ${ }^{2} \mathrm{~J}$ Gerstoft, 'S Cowan, 'S Hoffmann, $1 \mathrm{~J}$ $\mathrm{S}$ Jensen. 'Statens Serum Institut, Copenhagen, Denmark; ${ }^{2}$ Department of Infectious Diseases, Rigshospitalet, Copenhagen, Denmark

Background Since 1999, the number of persons diagnosed with syphilis has increased dramatically in Denmark. Molecular typing was used to investigate the epidemiology of Treponema pallidum aiming to understand the dynamics of the epidemic. In recent years the tp0548 gene sequence has been used to further differentiate the subtypes obtained using the CDC typing system (number of 60-base pair 